\title{
ESTIMATIVA DOS CORTES DIANTEIRO, COSTILHAR E SERROTE A PARTIR DO PESO DA CARCAÇA E DA CONFORMAÇÃO EM NOVILHOS HEREFORD
}

\author{
JARDIM, Pedro O. da C.; OSÓRIO, José C. da S. \& TAROUCO, Jaime U. \\ UFPEL/FAEM - Deptº de Zootecnia - Campus Universitário - Caixa Postal, 354 CEP 96010-900 - Tel (0532) 757270 - \\ Pelotas/RS - Brasil \\ (Recebido para publicação em 06/10/94)
}

\section{RESUMO}

O trabalho avalia o uso de medidas de fácil obtenção (conformação e peso de carcaça) como estimadores de peso dos cortes bovinos: serrote, costilhar e dianteiro. No experimento foram utilizados 58 carcaças de novilhos Hereford, criados em campo nativo e abatidos com 36 meses de idade. Os resultados, analisados estatísticamente, mostram que a conformação é um estimador pouco preciso do peso dos cortes serrote, costilhar e dianteiro. Já o peso da carcaça quente é uma medida mais precisa, devendo portanto ser incluída em um sistema de avaliação de carcaças.

Palavras-chave: carcaças, novilhos, Hereford, cortes da carcaça

\section{ABSTRACT}

This work studied the use of easy measurements (carcass conformation and carcass weight) as estimators of the weight of bovine cuts: forequarter, rib and hindquarter. Fifty eight Hereford steers carcasses were used in this experiment. The animals were raised in native pasture conditions and slaughtered with thirty six month of age. The results showed that conformation independent variable was poor estimator of the weight of cuts forequarter, rib and hindquarter. Already isolatedly warm carcass weight was the most precise measurement to estimate the weight of all cuts, therefore it must be included in a carcass evaluation system. carcass.

Key words: carcasses, steers, Hereford, cuts of the

\section{INTRODUÇÃO}

O sistema de tipificação procura utilizar medidas de fácil obtenção que realmente avaliem as diferenças em quantidade e qualidade existentes entre as carcaças .

Os pesos, vivo ou da carcaça, são utilizados pelos frigoríficos brasileiros para remunerar o produtor. $\mathrm{O}$ peso da carcaça e a conformação são medidas que estão incluídas no sistema de tipificação nacional de carcaças (BRASIL, 1990).

O peso vivo é uma medida objetiva, e segundo GUARENTI (1980), ZIEGLER (1980) e DODE (1984) apresenta uma elevada relação com o peso dos cortes serrote, costilhar e dianteiro quando não houver recorte de gordura.

A conformação é uma medida subjetiva que avalia fundamentalmente 0 desenvolvimento das massas musculares. MULLER (1980), refere-se a conformação como medida quantitativa, levando em consideração que animais de maior hipertrofia muscular proporcionam cortes com melhor aparência para o consumidor mais exigente, tendendo a apresentar menor proporção de osso e maior de porção comestível.

A relação existente entre a conformação e o peso dos cortes serrote, dianteiro e costilhar é positiva e significativa, segundo DODE (1984) e FARIAS et al (1986). Entretanto, JARDIM (1975), trabalhando com novilhos Aberdeen Angus, obteve um coeficiente de determinação não significativo entre a conformação e o peso do corte serrote.

Para verificar a possibilidade da utilização da conformação e do peso da carcaça, como estimadores do peso dos cortes dianteiro, costilhar e serrote, em novilhos Hereford, foi realizado o presente trabalho.

\section{MATERIAL E MÉTODOS}

\section{Animais}

O experimento utiliza carcaças de 58 novilhos da raça Hereford, com aproximadamente 36 meses de idade, criados exclusivamente em pastagem natural e que foram abatidos no Frigorífico Extremo Sul, Pelotas-RS, após um período de jejum de 24 horas, sob inspeção federal. As carcaças foram seccionadas no sentido longitudinal, seguindo uma linha no centro da coluna vertebral, sendo resfriadas por um período de 24 horas, a temperatura de ${ }^{\circ}{ }^{\circ} \mathrm{C}$, após o qual foram avaliadas e cortadas. 
JARDIM et al. Carcaças de Novilhos Hereford

\section{Peso da carcaça quente}

O Peso da carcaça foi tomado logo após o abate, antes de entrar na câmara de esfriamente.

\section{Conformação}

Para a medida subjetiva da conformação, foi seguido o sistema sugerido por MULLER (1980).

Peso do serrote, costilhar e dianteiro

Todos os cortes foram obtidos da meia carcaça direita, seguindo o precedimento normal do frigorífico Extremo Sul. (Pelotas/RS).

\section{Estatística}

Foram realizadas equações de regressão linear simples e multipla e calculados os desvios padrão residual (KEMPSTER et al, 1982). O peso da carcaça quente (PCQ) e a conformação(C) foram utilizados como variáveis independentes para estimar o peso do serrote (PS), do costilhar (PC) e do dianteiro (PD). O programa utilizado foi o ABSTAT (ANDERSON - BELL, 1984).

\section{RESULTADOS E DISCUSSÃO}

$\mathrm{Na}$ Tabela 1, são apresentadas as estatísticas descritivas das medidas realizadas.

TABELA 1 - Estatística descritiva das características da carcaça de novilho Hereford

\begin{tabular}{ccccc}
\hline CARACTERISTICAS & UNIDADE & MÉDIA & $\begin{array}{c}\text { DESVIO } \\
\text { PADRÃO }\end{array}$ & AMPLITUDE \\
\hline PCQ & $\mathrm{kg}$ & 187,431 & 19,46 & $151,0-224,0$ \\
$\mathrm{C}$ & índice & 6,603 & 1,68 & $4,0-10,0$ \\
$\mathrm{PS}_{\mathrm{a}}$ & $\mathrm{kg}$ & 42,714 & 3,96 & $35,6-51,2$ \\
$\mathrm{PC}_{\mathrm{a}}$ & $\mathrm{kg}$ & 12,431 & 2,00 & $7,8-16,8$ \\
$\mathrm{PD}_{\mathrm{a}}$ & $\mathrm{kg}$ & 36,376 & 3,84 & $28,4-43,2$ \\
\hline
\end{tabular}

a Medidas obtidas na meia carcaça direita.

$\mathrm{Na}$ Tabela 2, estão as equações para estimar o peso do serrote.

A Equação 1, mostra que o peso da carcaça quente foi a variável que apresentou maior influência no peso do serrote, explicando $94,92 \%(P=0,0000)$ da variação total no peso do corte. O coeficiente de regressão foi de $0,198 \mathrm{~kg}$, com um desvio padrão residual de $0,901 \mathrm{~kg}$.

Trabalhos realizados por JARDIM (1975); MULLER \& BORGES (1976); OSÓRIO et al. (1983a) e DODE (1984), mostram que peso de carcaça foi responsável por $85,32 \%$; $96,00 \% ; 96,00 \%$ e $95,00 \%$ da variação total no peso do serrote, respectivamente. Os coeficientes de determinação são elevados como os do presente trabalho; o que difere são os coeficientes de regressão, provavelmente pela utilização de raças diferentes, porque conforme BERG \& BUTTERFIELD (1966), existe diferença significativa entre raças no aumento do músculo em relação ao incremento no peso de carcaça.

A conformação, isoladamente (Equação 2), explicou $31,62 \%(P=0,0000)$ da variação total no peso do serrote. $O$ coeficiente de regressão foi de 1,32 e o desvio padrão residual foi de $3,308 \mathrm{~kg}$.

GUARENTI (1980) e ZIEGLER (1980), verificaram que a conformação explicou $29,44 \%$ e $41,19 \%$ da variação total do peso do serrote. Entretanto, JARDIM (1975), encontrou que a conformação da carcaça foi responsável por somente $8,30 \%(P>0,05)$ da variação no peso do corte serrote, sendo o peso da carcaça a principal fonte de variação no peso do serrote, pois as carcaças de melhor conformação foram também as mais pesadas.

As carcaças melhor conformadas apresentaram maior peso do corte serrote, porém, essa relação não foi tão elevada para justificar o uso dessa medida na estimativa do peso do serrote.

O acréscimo da conformação como variável independente (Equação 3) aumentou apenas 0,02\% o coeficiente de determinação, não justificando sua inclusão. Esse resultado concorda com os obtidos por GUARENTI (1980) e ZIEGLER (1980).

A Equação 4, mostra que a variável de maior influência no peso do costilhar é o peso da carcaça quente, explicando $79,45 \% \quad(P=0,0000)$ da variação do referido corte. O coeficiente de regressão foi de $0,029 \mathrm{~kg}$ e o desvio padrão residual de $0,918 \mathrm{~kg}$. Resultados similares foram obtidos por ZIEGLER (1980), OSÓRIO et al. (1983b) e FARIAS et al. (1986), que, trabalhando com novilhos, encontraram coeficientes de determinação $(P<0,01)$ de, respectivamente, $80,97 \% ; 77,00 \%$ e $71,00 \%$ entre o peso de carcaça e o peso do costilhar.

A Equação 5, mostra que a conformação isoladamente explicou $34,41 \% \quad(P=0,0000)$ da variação total do peso do corte costilhar. O coeficiente de regressão foi de $0,699 \mathrm{~kg}$, com um desvio padrão residual de 1,640 $\mathrm{kg}$. 
TABELA 2 - Equações de regressão para estimar o peso do serrote (PS), costilhar (PC) e dianteiro (PD)

\begin{tabular}{|c|c|c|c|}
\hline EQUAÇÃO DE REGRESSÃO & SIGNIF. & $\mathrm{r} 2 \times 100$ & D.P.R. \\
\hline $\begin{array}{c}\text { 1. } \mathrm{PS}=5,51+0,198(\mathrm{PCQ}) \\
32,36 \\
(\mathrm{P}=0,0000)\end{array}$ & $P=0,000$ & 94,92 & 0,901 \\
\hline 2. $\begin{aligned} P S=33,97+1,323(C) \\
5,08 \\
(P=0,0000)\end{aligned}$ & $P=0,000$ & 31,62 & 3,308 \\
\hline 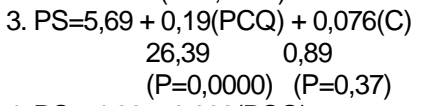 & $P=0,000$ & 94,99 & 0,903 \\
\hline 4. $\begin{aligned} & P C=-4,80+0,092(P C Q) \\
& 14,71 \\
&(P=0,0000)\end{aligned}$ & $P=0,000$ & 79,45 & 0,918 \\
\hline 5. $\begin{array}{c}\mathrm{PC}=7,82+0,699(\mathrm{C}) \\
5,42\end{array}$ & $P=0,000$ & 34,41 & 1,640 \\
\hline $\begin{array}{c}(\mathrm{P}=0,0000) \\
\text { 6. } \mathrm{PC}=-4,42+0,08(\mathrm{PCQ})+0,59(\mathrm{C})\end{array}$ & $P=0,000$ & 80,69 & 0,898 \\
\hline $\begin{array}{ll}11,48 & 1,88 \\
(P=0,0000) & (P=0,065)\end{array}$ & $P=0,000$ & & \\
\hline $\begin{array}{c}\text { 7. } P D=1,09+0,188(P C Q) \\
23,84 \\
(P=0,0000)\end{array}$ & $P=0,000$ & 91,03 & 1,160 \\
\hline $\begin{array}{r}\text { 8. } \mathrm{PD}=29.85+0,986(\mathrm{C}) \\
\qquad \begin{array}{l}3,60 \\
(\mathrm{P}=0,0000)\end{array}\end{array}$ & $P=0,000$ & 18,82 & 3,491 \\
\hline 9. $\begin{array}{rr}P D=0,35+0,203(P C Q) & -0,312(C) \\
22,95 & 3,05 \\
(P=0,0000) & (P=0,003)\end{array}$ & & 92,33 & 1,083 \\
\hline
\end{tabular}

* Os números logo abaixo dos coeficientes de regressão são os valores calculados de T e abaixo destes o nível de significância.

D.P.R. = desvio padrão residual da equação de regressão.

Resultado similar foi obtido por ZIEGLER (1980), onde a conformação foi responsável por 29,38\% $(P<0,01)$ da variação total do peso do costilhar. Entretanto, LAUZER et al. (1979) obtiveram resultados que demonstraram que o peso do corte costilhar não foi significativamente influenciado pela conformação, e que a causa foi o fato das carcaças de pior conformação apresentarem maior peso, devido a maior espessura de gordura de cobertura. GUARENTI (1980), confirma os resultados em que a conformação não influenciou significativamente a variação no peso do costilhar.

A conformação, apesar da significância estatística, não foi um estimador preciso na predição do peso do costilhar, apresentando um desvio padrão residual bem mais elevado que quando foi utilizado o peso da carcaça quente (Equação 4).

Quando na equação a conformação (Equação 6), foi acrescentada ao peso de carcaça como variável independente, o aumento foi de apenas 1,24\% no coeficiente de determinação, não justificando sua inclusão, concordando com os resultados encontrados por GUARENTI (1980) e ZIEGLER (1980).

O peso de carcaça quente, isoladamente (equação 7), explicou $91,03 \%(\mathrm{P}=0,0000)$ da variação total do peso do corte dianteiro. O coeficiente de regressão foi de $0,188 \mathrm{~kg}$ e um desvio padrão residual de $1,160 \mathrm{~kg}$. Resultados semelhantes foram obtidos por ZIEGLER (1980); OSÓRIO et al. (1983a); DODE (1984) e FARIAS et al. (1986), onde o peso da carcaça explicou 89,91\%; 97,00\%; 95,00\% e $97,00 \%$ na variação do peso do dianteiro, respectivamente.

A tendência do presente estudo foi similar à dos trabalhos revisados. As diferenças encontradas foram devidas à quantidade de ganho de peso do corte dianteiro em relação ao ganho de peso de carcaça. A explicação para essa resposta pode estar na afirmação de NOUR et al. (1983), de que o valor absoluto de uma característica de carcaça em qualquer peso dado é determinada pela raça (principalmente refletindo diferenças no tamanho adulto) e por diferenças de manejo.

A conformação (Equação 8) explicou 18,82\% $(\mathrm{P}=0,0007)$ na variação total do peso do dianteiro. $\mathrm{O}$ coeficiente de regressão foi positivo, e o desvio padrão residual, de 3,491 kg. GUARENTI (1980), obteve resultado semelhante, tendo a conformação explicado $17,38 \%$ da variação total no peso do dianteiro. Coeficientes de determinação mais elevados foram obtidos por ZIEGLER $(1980)$ e FARIAS et al. (1986), com valores de 38,26 e 57,00 , respectivamente.

Os trabalhos revisados demonstram uma variação nos resultados, o que pode ser explicado pela afirmação de FORREST et al (1979), de que a medida que o animal cresce e se desenvolve, desde o nascimento até a 
maturidade, seu organismo sofre modificações continuas em connformação e composição, e dentro da mesma espécie os animais que na maturidade tem maior tamanho necessitam, para cada fase de crescimento, períodos de tempo maiores do que os exigidos por animais cujo tamanho é menor na maturidade.

Quando foi acrescentada a conformação como variável independente, (Equação 9), houve um aumento no coeficiente de determinação de $1,3 \%$, indicando pequeno aumento na precisão da estimativa do peso do dianteiro.

Resultado similares foram obtidos por GUARENTI (1980), onde o aumento do coeficiente de determinação foi de somente 0,07 e por ZIEGLER (1980), que utilizando as mesmas variáveis, verificou que o aumento no coeficiente de determinação foi de apenas $0,21 \%$ em relação ao peso de carcaça.

Os resultados obtidos no presente trabalho e os citados anteriormente, mostram que a inclusão da conformação como segunda variável independente na estimativa do peso do dianteiro, não representa um importante aumento no coeficiente de determinação.

\section{CONCLUSÕES}

A conformação é um estimador pouco preciso dos pesos do serrote, costilhar e dianteiro, não devendo ser utilizada isoladamente como índice quantitativo em um sistema de avaliação de carcaças.

O peso de carcaça quente, isoladamente, foi o estimador mais preciso do peso dos cortes, devendo ser utilizado num sistema de avaliação de carcaças.

\section{REFERÊNCIAS BIBLIOGRÁFICAS}

ANDERSON-BELL. Manual do ABSTAT 4.08 Canon City. Colorado, USA, 1984. $136 \mathrm{p}$.

BERG, R.T. \& BUTTERFIELD, R.M. Muscle: bone ratio and percentageas measures of beef carcass composition. Animal Production,v.8, n.1, p.1-11, 1966.

BRASIL. SECRETARIA NACIONAL DE DEFESA AGROPECUÁRIA; SECRETARIA DE INSPEÇÃO DE PRODUTO ANIMAL. Padronização dos cortes de carne bovina. Brasília. MA/SNDA/SIPA. 1990. 98 p.

DODE, M.A.M. Avaliação da produção de carne em carcaças de novilhos Holandês. Curso de PósGraduação em Zootecnia, Universidade Federal de Pelotas, Pelotas, RS, 1984, 73 p. (Tese de Mestrado)

FARIAS, J.V.S. ; JARDIM, P.O.C. ; GUERREIRO, J.L.V. ; OSÓRIO, J.C.S. Avaliação da produção de carnes em novilhos Hereford. II - Comparação do peso de carcaça e conformação como estimadores. In: 23 $3^{\underline{a}}$ REUNIÃO ANUAL DA SOCIEDADE BRASILEIRA DE ZOOTECNIA, 1986. Campo Grande, MS. Anais... Campo Grande, SBZ, 1986. p.186.

FORREST, J. C. ; ABERLE, E. D. ; HEDRICK, H. B. ; JUDGE, M. D. ; MERKEL, R. A. Fundamentos de ciencia de la carne. Editorial Acribia, Zaragoza, Espanã, 1979. 364 p.

GUARENTI, N.M. Avaliação da carcaça de vacas de descarte. Curso de Pós-Graduação em Zootecnia, Universidade Federal de Pelotas, Pelotas, RS, 1980, 101 p. (Tese de Mestrado)

JARDIM, P.O.C. Efeito da conformação e do peso de carcaça quente no rendimento da porção comestível da carcaça bovina. Curso de Pós-Graduação em Zootecnia, Universidade Federal de Santa Maria, Santa Maria, RS, 1975.78 p. (Tese Mestrado)

KEMPSTER, A.J. ; CUTHBERTSON, A. ; HARRIGTON, $G$. The relationship between conformation and the yield and distribution of lean mean in the carcasses of British pigs, cattle and sheep: A Review. Meat Science, v.5, p.37-53, 1982.

LAUZER, J. J. ; MULLER, L. ; SILVA, S.F. da. A influência da conformação no rendimento de alguns cortes da carcaça bovina. Revista da Sociedade Brasileira de Zootecnia, v.8, n.1,p.102-109, 1979.

MULLER, L. \& BORGES, F.V. Rendimento do "Corte serrote" em novilhos de corte. In: 13a REUNIÃO ANUAL DA SOCIEDADE BRASILEIRA DE ZOOTECNIA. 1976. Salvador, BA. Anais... Salvador, SBZ, 1976. p. 114.

MULLER, L. Normas para avaliação de carcaças e concurso de carcaças de novilhos. Santa Maria. Departamento de Zootecnia- UFSM-RS. 1980. 31 p. (Publicação, 1).

NOUR, A. Y. M. ; THONNEY, M. L. ; STOUFFERR WHITE, W. R. C. Jr. Changes in carcass weight and characteristics with increasing weight of large and small cattle. Journal of Animal Science, v.57, n.5, p.115465, 1983.

OSÓRIO, J.C.S. ; GUERREIRO, J.L.V. ; ZONTA, E.P. ; MACHADO, A.A. ; CONY, C.A.O. Determinação do peso de carcaça em novilhos. In: ENCONTRO DE PESQUISA VETERINÁRIA. 1983. Pelotas, RS. Anais... Pelotas, UFPEL, 1983a, p.2.

OSÓRIO, J.C.S. ; JARDIM, P.O.C. ; GUERREIRO, J.L.V. ; FARIAS, J.V.S.; CONY, C.A.; ZONTA, E.P. Produção de carne de bovinos abatidos em frigorífico: 2. Predição dos cortes dianteiro, costilhar e serrote. Pelotas, EMBRAPA, 1983b. (Comunicado Técnico, 13).

ZIEGLER, J.C. Avaliação da produtividade de carcaças de novilhos. Curso de Pós-Graduação em Zootecnia, Universidade Federal de Pelotas, Pelotas, RS, 1980. 115 p. (Tese de Mestrado) 